\title{
Neutrophil Lymphocyte Ratio to Albumin Ratio and White Blood Cell to Hemoglobin Ratio as Prognostic Markers for Hepatocellular Carcinoma Patients Who Underwent Curative Hepatectomy
}

\author{
Xueqin Shen $\mathbb{D}^{*}$ \\ Wei Wang (iD* \\ Xiaoping Niu (D) \\ Department of Gastroenterology, \\ Yijishan Hospital of Wannan Medical \\ College, Wuhu, Anhui Province, People's \\ Republic of China \\ *These authors contributed equally to \\ this work
}

Purpose: Inflammation factors affect the prognosis of hepatocellular carcinoma (HCC). However, it is not clear if the preoperative neutrophil lymphocyte ratio to albumin ratio (NLRAR) and white blood cell to hemoglobin ratio (WHR) affect the prognosis of HCC post curative hepatectomy. We assessed the prognostic values of NLRAR and WHR in patients suffering from HCC who underwent curative resection.

Materials and Methods: A total of 169 eligible HCC cases were reviewed. The optimal cut-off values for NLRAR and WHR were selected using the X-tile software. The overall survival (OS) rate was assessed following the Kaplan-Meier analysis method. The Log rank test and Cox proportional hazard regression model were also used to analyze the data. The prognostic values of NLRAR and WHR were calculated by analyzing the area under the receiver operating characteristic curve (AUC). The decision curve analysis (DCA) was performed for clinical benefits.

Results: The OS rate recorded for the high NLRAR group was poorer than the OS rate recorded for the low NLRAR group. Similar trends were observed for WHR. The NLRAR and WHR were the independent predictors of OS. The results were based on the multivariate Cox analyses method. Results obtained by analyzing the subgroups revealed that NLRAR and WHR could be used for the prognosis of HCC in tumor stage I-patients. The NLRAR-WHR scoring system (NWS) could be used to classify HCC patients into two cohorts with different prognoses. This scoring system was more efficient than NLRAR or WHR in predicting OS.

Conclusion: The preoperative NLRAR and WHR are effective prognostic indicators for HCC in patients who underwent curative hepatectomy.

Keywords: hepatocellular carcinoma, neutrophil lymphocyte ratio to albumin ratio, white blood cell to hemoglobin ratio, prognosis, overall survival, curative hepatectomy

\section{Introduction}

Liver cancer is the fourth leading cause of cancer-related deaths in the world. More than 780,000 people in the world die of liver cancer annually. ${ }^{1}$ Hepatocellular carcinoma (HCC) is the most common type of primary liver cancer and accounts for $75-85 \%$ of the total liver cancer cases reported worldwide. ${ }^{1}$ Curative hepatectomy remains the mainstay of treatment modality for patients suffering from $\mathrm{HCC}^{2}$ Unfortunately, the outcome of HCC patients who underwent curative hepatectomy remain unsatisfactory. 2,3
Correspondence: Xiaoping Niu Department of Gastroenterology, Yijishan Hospital of Wannan Medical College,

No. 2, Zheshan Road, Wuhu, Anhui Province, 241000, People's Republic of China

Tel +86-0553-5739316

Email niuxiaoping135@163.com 


\section{Graphical Abstract}

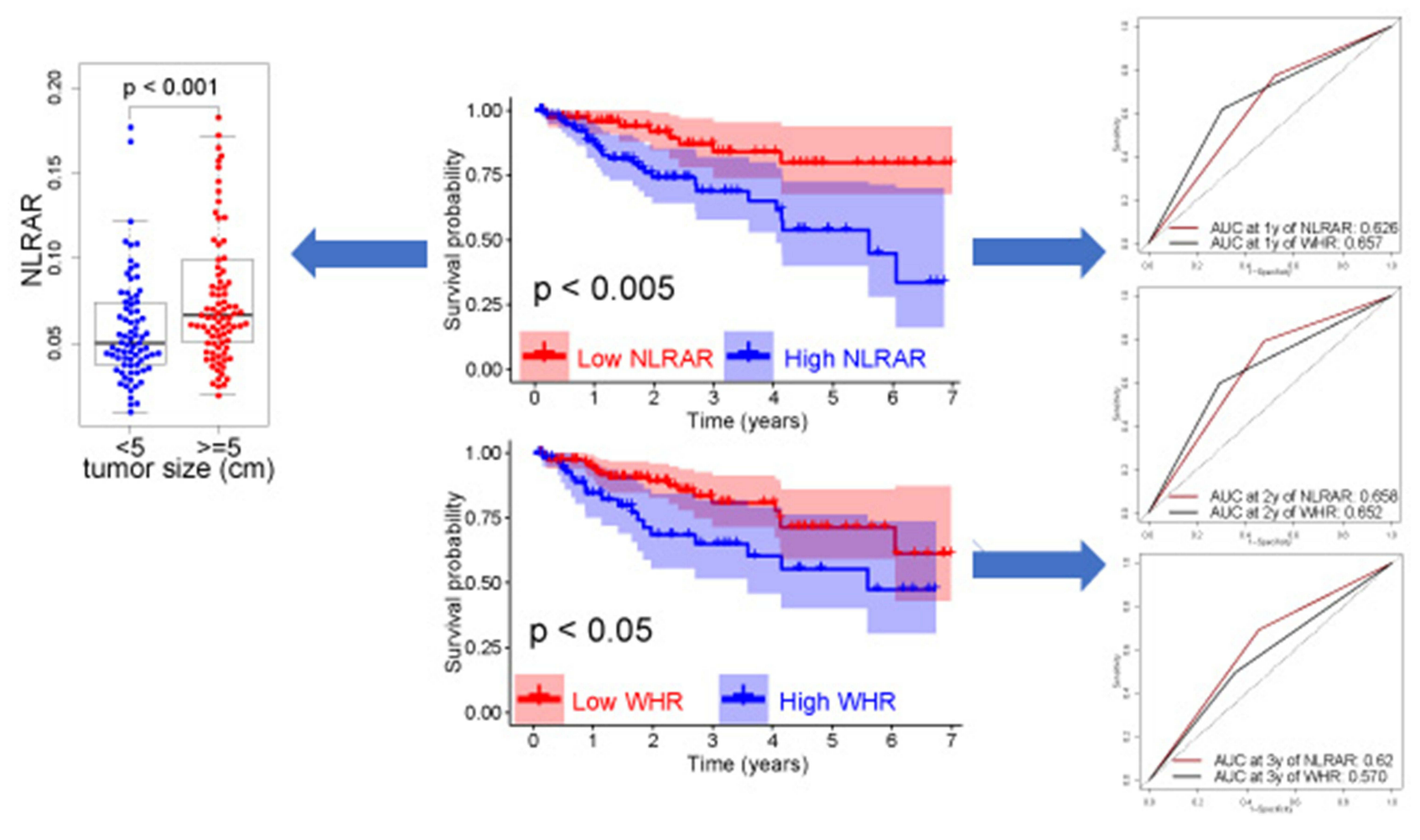

Systemic inflammation influences cancer development and progression and is an independent predictor of oncological prognosis. ${ }^{4,5}$ Some classical inflammation markers derived from blood samples, such as the lymphocyte-to-monocyte ratio (LMR), neutrophil-to-lymphocyte ratio (NLR), and platelet-to-lymphocyte ratio (PLR), have been identified as prognostic markers in several types of tumors, including HCC. ${ }^{6-12}$ It has been recently reported that the neutrophil lymphocyte ratio to albumin ratio (NLRAR) and white blood cell to hemoglobin ratio (WHR) are survival predictors of patients suffering from esophageal squamous cell carcinoma and gastric adenocarcinoma, respectively. ${ }^{13,14}$ However, the prognostic values of NLRAR and WHR in patients suffering from HCC and subjected to curative resection are unclear. Therefore, the purpose of this study was to investigate the ability of NLRAR and WHR in predicting the survival rate of patients suffering from HCC post curative hepatectomy.

\section{Materials and Methods}

\section{Patients}

We reviewed the consecutive primary HCC patients who underwent curative hepatectomy between March 2012 and June 2019 at the Yijishan Hospital of Wannan Medical
College. The exclusion criteria were determined: (1) not first hepatectomy, (2) multiple primary malignancies, (3) distant organ metastasis, (4) treatment with neoadjuvant therapy, (5) diagnosed combination with acute inflammatory disease, autoimmune disease, or haematopoietic system disease, (6) incomplete clinicopathological information, (7) survival time $<1$ month. A total of 169 eligible HCC patients were enrolled. After the patients were discharged, blood tests and abdomen image scan including abdominal ultrasonography, contrastenhanced computed tomography (CT) or magnetic resonance imaging (MRI) were regularly performed every three to six months during the first two years. Following this, patients were followed up and received serum and imaging examination every six months. The final follow-up time was December 5th 2019. The overall survival (OS) time was defined as the interval between the date of surgery and the time of patient death (event) or the last follow-up time. All patients suffering from HCC were staged following the American Joint Committee on Cancer (AJCC) 8th staging system. ${ }^{15}$

\section{Data Collection}

Blood samples were obtained prior to surgery within one week. Clinicopathological data such as age at 
diagnosis, gender, surgical procedure, tumor size, and number of tumor nodules were also collected. The NLR was defined as the absolute neutrophil count divided by the absolute lymphocyte count. NLRAR was defined as the NLR divided by the albumin (Alb). The WHR was calculated as the white blood cell (WBC) count divided by the hemoglobin (HB) level. The optimal cut-off values of NLRAR and WHR were determined using the X-tile software. ${ }^{16}$ The patients with values above the cut-off values were considered to be in the high group, while the patients with values below the cut-off values were considered to be in the low group. An NLRAR-WHR scoring system (NWS) was constructed to understand the combined predictive effects of NLRAR and WHR on the prognosis of patients suffering from HCC: patients with low NLRAR and low WHR were assigned a score of 0 . Those with high NLRAR and high WHR were assigned a score of 2 . The patients with a high NLRAR or a high WHR were assigned a score of 1 . The patients with a score of 0 or 1 (low NWS) were assigned to the low-risk group, and the patients with a score of 2 (high NWS) were assigned to the high-risk group.

\section{Statistical Analysis}

Categorical and continuous variables were indicated as frequency (percentage) and median (range), respectively. The OS rates were analyzed using the KaplanMeier (KM) method, and compared using the Log rank test. The independent risk factors associated with HCC survival were determined using the multivariate Cox regression analysis. The predictive powers of NLRAR, WHR, and NWS were determined by analyzing the area under the receiver operating characteristic (AUC) curve (ROC). The net benefits of using NLRAR, WHR, and NWS were determined following the decision curve analyses (DCA). The statistical analyses were performed using the $\mathrm{R}$ (version 3.6.1). A P-value of $<0.05$ in two-tailed tests indicated a statistically significant difference.

\section{Results}

\section{Patient Characteristics}

The median age of the enrolled patients was $61(26-85)$ years, and the median follow-up time was 23.5 (1-85) months. The 1-, 2- and 3-year OS rates for patients suffering from HCC who underwent curative
Table I Clinicopathological Variables of the Enrolled Patients

\begin{tabular}{|c|c|}
\hline Variables & $N=169$ \\
\hline Sex (Male/Female) & I39/30 (82.2/17.8) \\
\hline Age (years, $\leq 60 />60$ ) & $80 / 89(47.3 / 52.7)$ \\
\hline $\operatorname{AFP}(\mathrm{ng} / \mathrm{mL},<400 / \geq 400)$ & II5/54 (68.0/42.0) \\
\hline PLT $\left(\times 10^{9} / \mathrm{L}\right)$ & $(127,7-355)$ \\
\hline ALB $(g / L,<35 / \geq 35)$ & $36 / 133(2 \mid .3 / 78.7)$ \\
\hline ALT (U/L, <40/ $\geq 40)$ & III/58 (65.7/34.3) \\
\hline 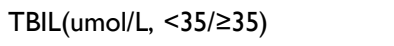 & I57/I2 (92.9/7.1) \\
\hline Tumor size $(\mathrm{cm},<5 / \geq 5)$ & $78 / 91(46.2 / 53.8)$ \\
\hline Tumor number (solitary/multiple) & $139 / 30(82.2 / 17.8)$ \\
\hline Tumor stage $(I / I-I I I)$ & $106 / 63(62.7 / 37.3)$ \\
\hline Surgical procedure (AR/NAR) & II $2 / 57(66.3 / 33.7)$ \\
\hline NLRAR & $(0.0600,0.0095-1.2043)$ \\
\hline WHR & $(4.07,1.59-12.07)$ \\
\hline NWS (low risk/high risk) & I24/45 (73.4/26.6) \\
\hline
\end{tabular}

Note: Values are presented as the median (range) or $\mathrm{n}(\%)$

Abbreviations: AFP, alpha-fetoprotein; ALB, albumin; PLT, platelet; ALT, alanine aminotransferase; TBIL, total bilirubin; AR, Anatomic resection; NAR, nonanatomic resection; NLRAR, neutrophil lymphocyte ratio to albumin ratio; WHR, white blood cell to hemoglobin ratio; NWS: NLRAR-WHR scoring system.

hepatectomy were $91.5 \%, 82.1 \%$, and $73.8 \%$, respectively. They were predominantly males, were subjected to anatomic resection, in tumor stage I, with a tumor size $\geq 5 \mathrm{~cm}$ and had single tumor nodule. The alpha fetoprotein (AFP) level in less than half of them $(41.6 \%)$ was $<400 \mathrm{ng} / \mathrm{mL}$. The detailed baseline information is presented in Table 1 .

\section{Determination of Cut-Off Values for the Inflammation Markers}

The results obtained using the X-tile software revealed that the optimal cut-off values for preoperative NLRAR and WHR were 0.056 and 4.8, respectively (Figure 1). Based on the cut-off values, the patients were assigned an NWS score and categorized into the low- and high-risk groups. Results from the KM analyses revealed that the 3-year OS rates recorded for patients with high NLRAR were significantly lower than the 3-year OS rate recorded for patients with low NLRAR $(68.7 \%$ and $83.9 \%$, respectively; $\mathrm{P}<$ 0.005) (Figure 2A). Similarly, the 3-year OS rates recorded for patients with high WHR were significantly lower than the 3-year OS rates recorded for patients with low WHR (65.0\% and 80.9\%, respectively; $\mathrm{P}<0.005)$ (Figure 2B). Furthermore, the 3-year OS rates recorded for the low-risk patients $(82.5 \%)$ were better than that recorded for the high-risk patients $(54.2 \%$; $\mathrm{P}<0.001)$ (Figure 2C). 

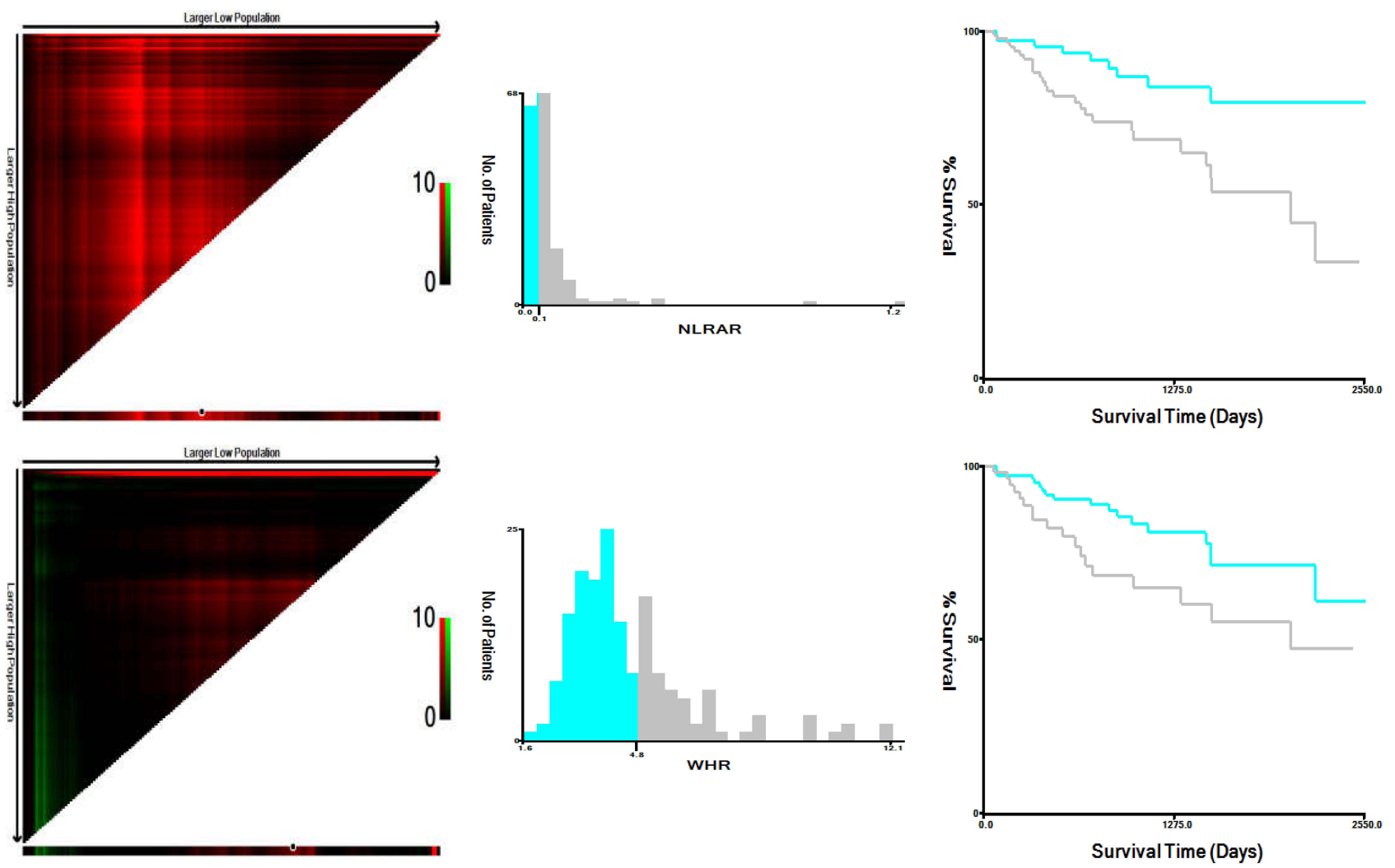

Figure I Cut-off values identified using the X-tile software.
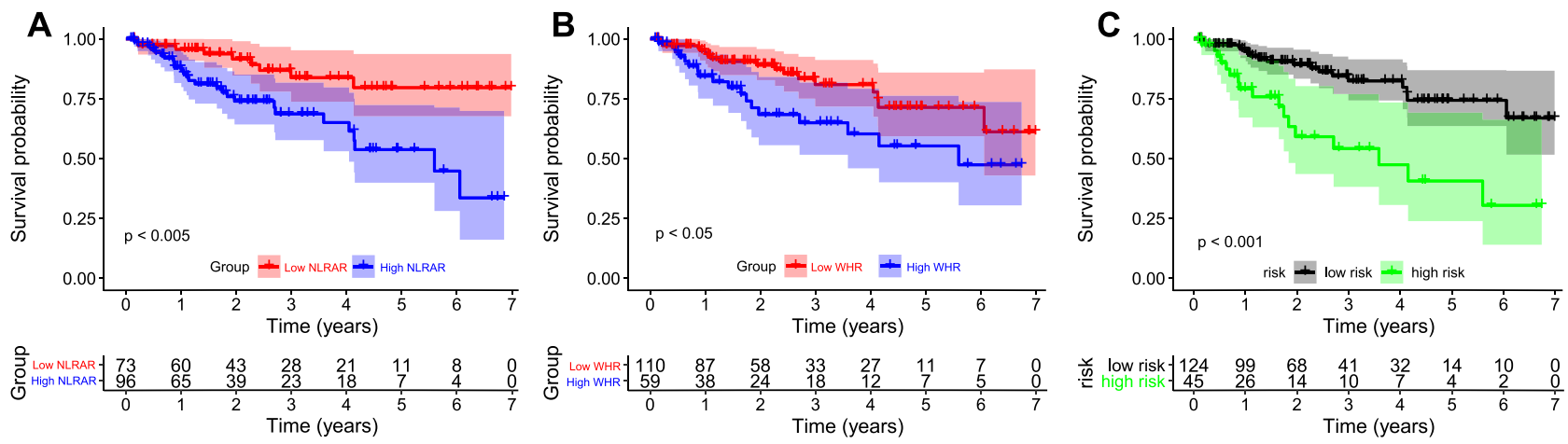

Figure 2 Kaplan-Meier survival curves plotted using data collected from HCC patients undergoing hepatectomy for overall survival. (A) Overall survival rates stratified by NLRAR. (B) Overall survival rates stratified by WHR. (C) Overall survival rates stratified by NWS.

Abbreviations: HCC, hepatocellular carcinoma; NLRAR, neutrophil lymphocyte ratio to albumin ratio; WHR, white blood cell to hemoglobin ratio; NWS: NLRAR-WHR scoring system.

\section{Univariate and Multivariate Cox Analyses of Potential OS Predictors}

The results from univariate analyses revealed that NLRAR, WHR, and NWS significantly influenced the OS rates of patients suffering from HCC. Furthermore, the results from multivariate analyses revealed that NLRAR, WHR, and NWS were independent prognostic predictors after adjusting for clinicopathological and blood biochemical variables (Table 2). The AUC values for predicting the 1-, 2- and 3-year OS rates of NWS were 0.705 , 0.690 , and 0.632 , respectively; of NLRAR were 0.626 , 0.658 , and 0.620 , respectively; and of WHR were 0.657 , 0.652 , and 0.570 , respectively (Figure $3 \mathrm{~A}-\mathrm{C}$ ). In addition, results obtained using the DCA revealed that the net 
Table 2 Univariate and Multivariate Cox Analyses of Inflammation Markers for Overall Survival

\begin{tabular}{|l|c|c|c|c|c|c|}
\hline \multirow{2}{*}{ Inflammation Based Markers } & \multicolumn{3}{|c|}{ Univariate Analyses } & \multicolumn{3}{|c|}{ Multivariate Analyses } \\
\cline { 2 - 7 } & HR & $\mathbf{9 5 \%} \mathbf{~ C l}$ & $\mathbf{P}$ & HR & $\mathbf{9 5 \%} \mathbf{~ I ~}$ & $\mathbf{P}$ \\
\hline NLRAR (elevated/decreased) & 3.05 & $1.43-6.50$ & 0.004 & $3.0 I^{*}$ & $1.36-6.65$ & $<0.01$ \\
WHR (elevated/decreased) & 2.06 & $1.07-3.96$ & 0.03 & $2.40^{\#}$ & $1.17-4.93$ & $<0.05$ \\
NWS (high risk/low risk) & 3.35 & $1.74-6.45$ & $<0.001$ & $3.66^{*}$ & $1.77-7.59$ & $<0.001$ \\
\hline
\end{tabular}

Notes: *HR and $95 \% \mathrm{Cl}$ from multivariate Cox analysis after adjusting for Age, Sex, AFP, PLT, ALT, TBIL, Tumor size, Tumor number, Tumor stage and Surgical procedure. \#HR and $95 \% \mathrm{Cl}$ from multivariate Cox analysis after adjusting for Age, Sex, AFP, PLT, ALB, ALT, TBIL, Tumor size, Tumor number, Tumor stage and Surgical procedure. Abbreviations: NLRAR, neutrophil lymphocyte ratio to albumin ratio; WHR, white blood cell to hemoglobin ratio; NWS, NLRAR-WHR scoring system.

benefits obtained using NWS were better than those obtained using NLRAR or WHR (Figure 3D). These results revealed that these inflammation indicators could function as predictors of OS rates in patients suffering from HCC. It was hypothesized that NWS was the best indicator as it exhibited a good discrimination ability and
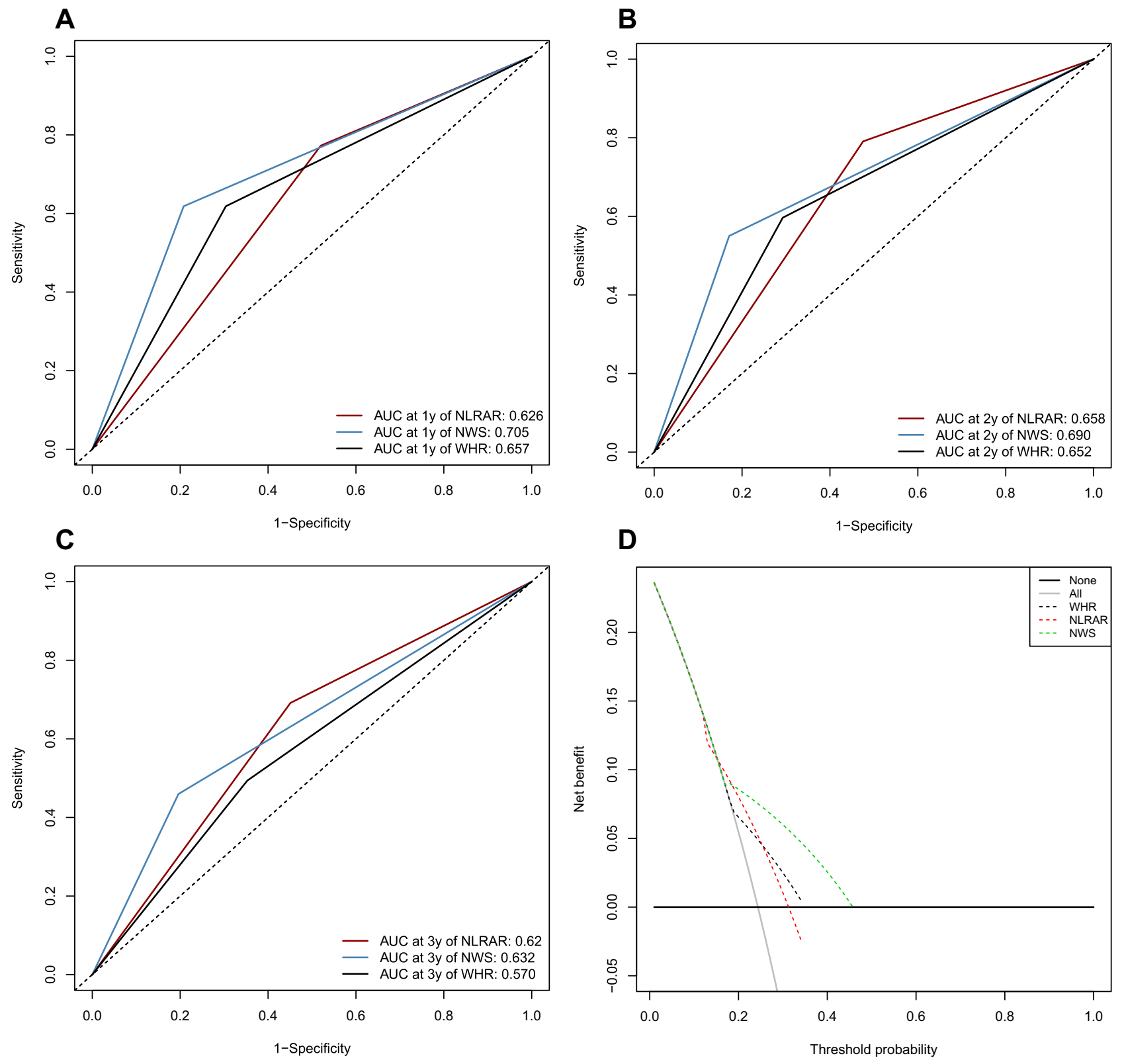

Figure 3 The predictive power of inflammation markers in overall survival rates for HCC patients undergoing hepatectomy. (A-C) Receiver operating characteristic curve analysis for inflammation markers for 1-, 2- and 3-year overall survival rates. (D) Decision curve analysis (DCA) for inflammation markers at clinical benefits. 
a comprehensive and actual range of threshold possibilities.

\section{Subgroup Analysis}

We conducted the subgroup analyses to investigate the NLRAR, WHR, and NWS performances for the prognosis in distinct tumor stage cohorts based on stratification of the total cohort according to different tumor stages. Results obtained using the KM analyses revealed that the OS rates recorded for patients with high NLRAR, WHR, and NWS were lower than the OS rates recorded for patients with low NLRAR, WHR, and NWS (tumor stage I-patients; Figure 4A-C). Significant differences in the OS rates were not observed between patients with high and low inflammation markers in tumor stages II-III (Figure 4D-F).
In the tumor stage I cohort, the ROC values of NLRAR at 1-, 2- and 3-year OS were 0.685, 0.700, and 0.627, respectively. The WHR at 1-, 2- and 3-year OS were $0.717,0.738$, and 0.612 , respectively, and the NWS at 1-, 2- and 3-year OS were 0.764, 0.763, and 0.672, respectively (Figure $4 \mathrm{G}-\mathrm{I}$ ). These findings suggested that NLRAR, WHR, and NWS could be used for the effective prognosis of early tumor stagepatients suffering from HCC who underwent curative resection.

\section{Correlations Between Inflammation Markers and Clinicopathological Features}

Following this, we investigated the relationship between the inflammation markers and the clinicopathological features. The results revealed that a low NLRAR level
A
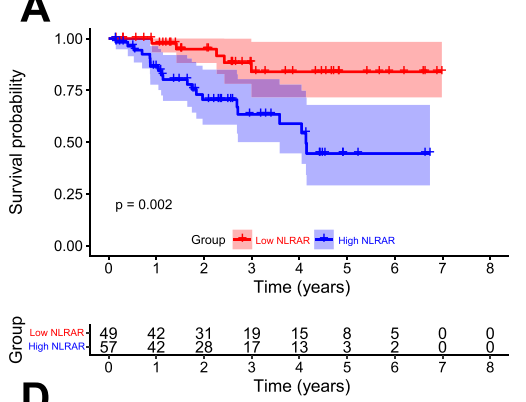

D
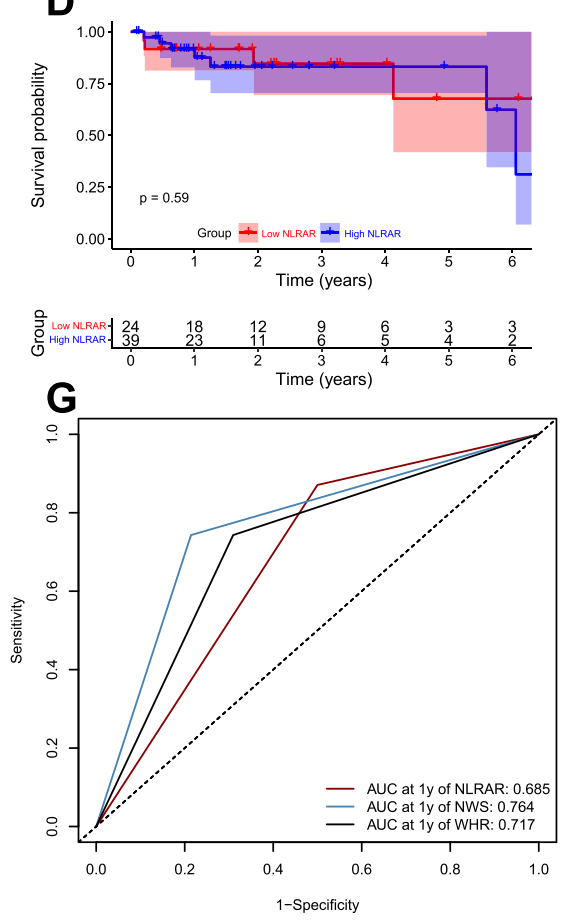

B
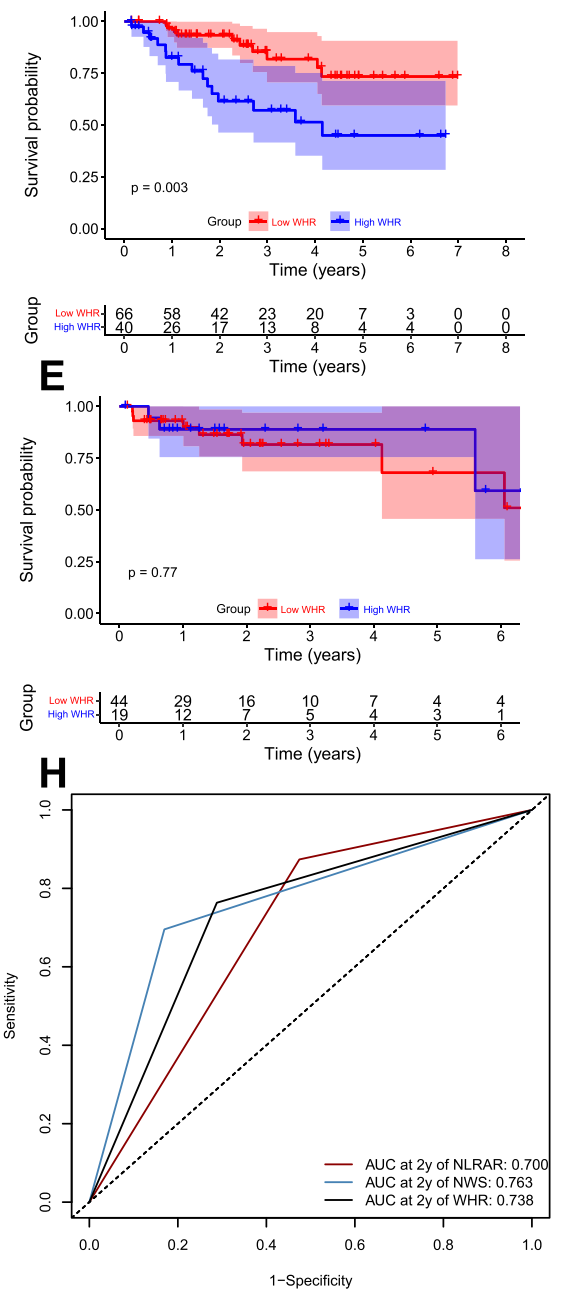
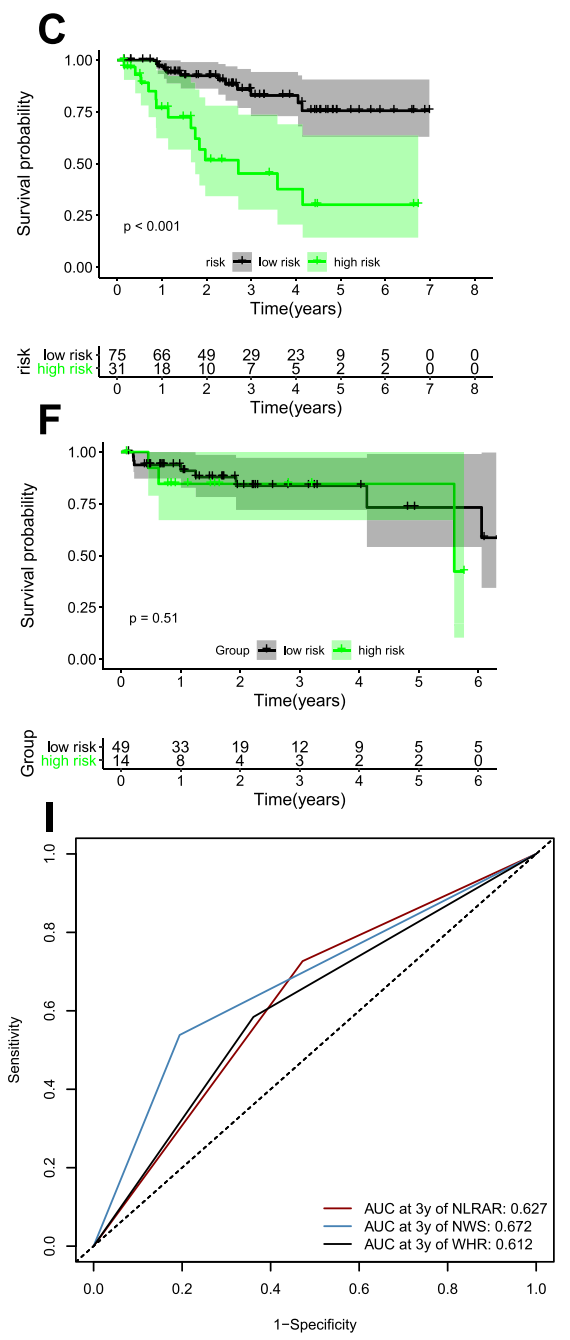

Figure 4 Subgroup analyses for inflammation markers in HCC patients undergoing hepatectomy stratified by different tumor stages. (A-C) Kaplan-Meier survival curves for overall survival rates at tumor stage I. (D-F) Kaplan-Meier survival curves for overall survival rates at tumor stages II-III. (G-I) Receiver operating characteristic curve analysis for the determination of I-, 2- and 3-year overall survival rates at tumor stage I. 
was significantly associated with small tumor size $(\mathrm{P}<$ $0.001)$ and a low total bilirubin (TBIL) level $(\mathrm{P}<0.05)$ (Figure 5). However, the WHR level could not be correlated with any clinicopathological or blood biochemical variables (data not shown). These results suggested that NLRAR could help identify the aggressive phenotype of the disease.

\section{Pearson Correlation}

Results from Pearson correlation analyses revealed a significant negative correlation between Alb and NLR $(r=-0.22, P<0.01$, Figure $6 \mathrm{~A})$ and a positive correlation between WHR and NLRAR $(\mathrm{r}=0.57, \mathrm{P}<0.001$, Figure 6B). However, no significant correlation was observed between WBC and HB (Figure 6C).

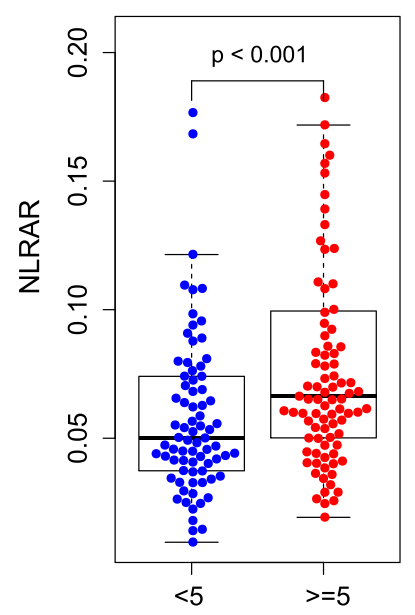

tumor size $(\mathrm{cm})$
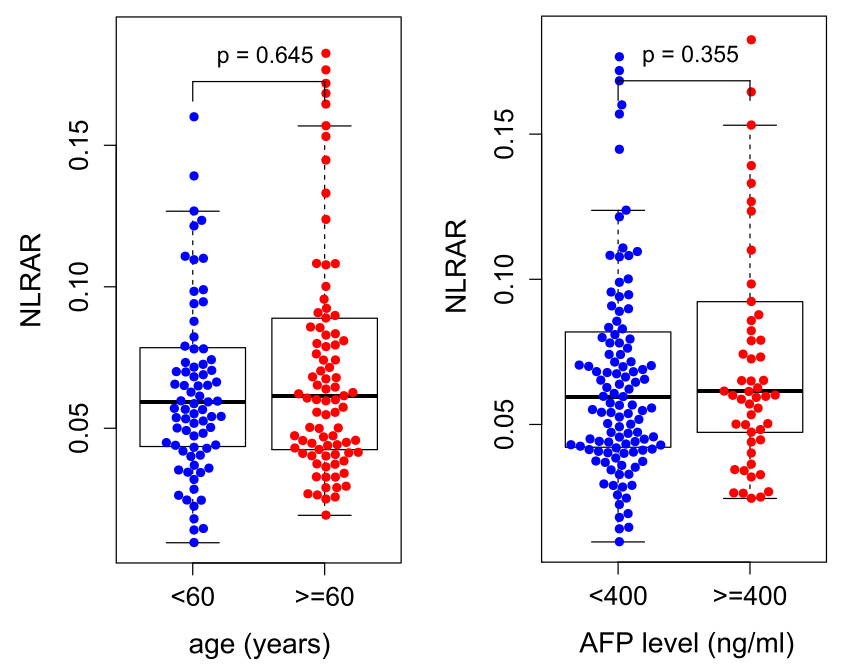

\section{Discussion}

It has been reported that chronic inflammation plays a crucial role in the initiation, development, and progression of HCC ${ }^{17}$ The predictive role of inflammation markers has been explored in recent years, and they have been used for the prognosis of patients suffering from HCC. To the best of our knowledge, we are the first to determine the abilities of preoperative NLRAR and WHR in predicting the prognosis for HCC patients who underwent curative hepatectomy.

We determined the optimal cut-off values for NLRAR and WHR using the X-tile analysis. The values were determined to be 0.056 and 4.8 , respectively. We observed that high levels of NLRAR and WHR were associated with poor OS. We also found
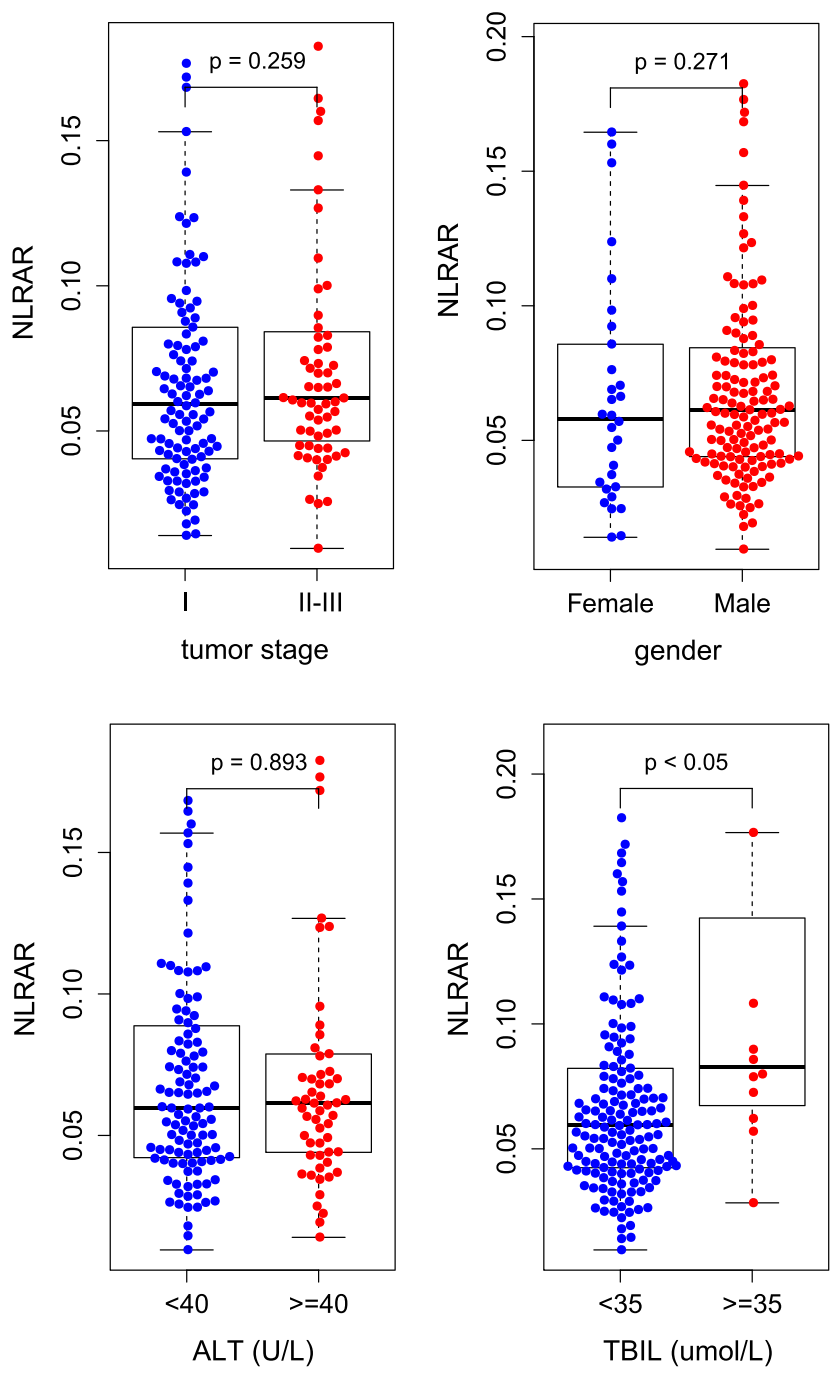

Figure 5 Correlations between inflammation markers and clinicopathological features. 

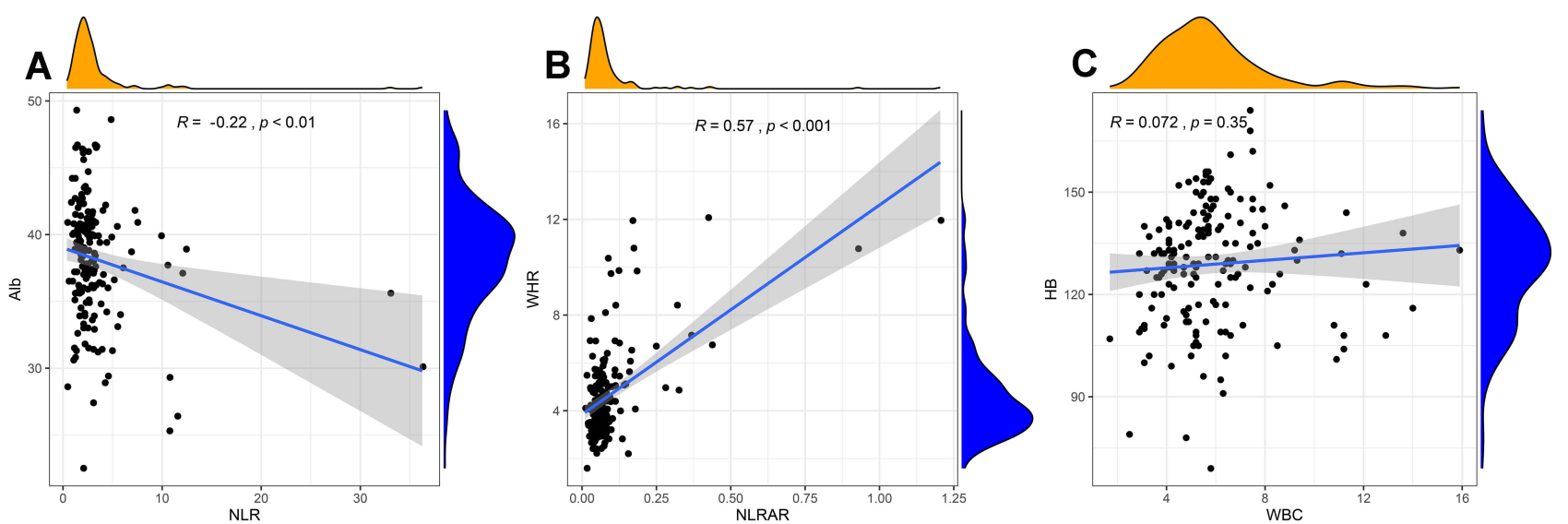

Figure 6 Pearson correlation analysis. (A) Negative correlation between albumin and NLR; (B) positive correlation between WHR and NLRAR. (C) No significant correlation was observed between hemoglobin and white blood cell.

Abbreviations: NLR, neutrophil lymphocyte ratio; NLRAR, neutrophil lymphocyte ratio to albumin ratio; WHR, white blood cell to hemoglobin ratio; NWS, NLRARWHR scoring system.

that preoperative NLRAR, WHR, and NWS were the independent prognostic factors for OS in HCC. Furthermore, we also observed that these inflammation markers significantly influenced the OS rates in the subgroup consisting of patients in whom the tumor(s) are in their early stages of development. The results from ROC analyses revealed that NLRAR and WHR could effectively predict the OS rates in patients suffering from HCC. Moreover, the predictive power of NWS was better than the predictive powers of NLRAR and WHR.

Zhao et al were the first to report that NLRAR was a useful predictive and prognosis factor for patients suffering from esophageal squamous cell carcinoma. ${ }^{13}$ It has been previously reported that NLR is an independent indicator of OS in patients suffering from HCC. ${ }^{18-20}$ High Alb levels reflected better outcomes in some liver diseases. ${ }^{21-23}$ In clinical practice, both NLR and Alb are influenced by various conditions. Therefore, the ratio of NLR to Alb could impair the potential basis to some extent. ${ }^{13}$ Based on these findings, we hypothesized that NLRAR could predict the prognosis of patients with HCC because it can potentially reflect the balance between systemic inflammation and trophic status.

Generally, elevated WBC and decreased HB levels indicate a compromised immune system. It has been previously reported that a low HB level was related to poor prognosis in various types of malignancies. ${ }^{24-27}$ In addition, results from an epidemiological study revealed that an increased WBC level was positively associated with cancer mortality. ${ }^{28}$ WHR exhibited a good prognostic value for patients suffering from gastric adenocarcinoma. ${ }^{14}$ Our results support the notion that WHR can independently predict the prognosis of HCC patients.

However, there are several drawbacks in this study. Firstly, it is a retrospective analysis conducted using data obtained from a single institution. This could result in selection bias. Therefore, the prognostic values of NLRAR and WHR need to be validated by analyzing a well-designed and large cohort. Secondly, we did not put some affecting factors for prognosis of HCC into consideration in this study, such as the effectiveness of surgery, the complications after surgery and adjuvant treatment.

\section{Conclusion}

In short, we supplied evidence that WHR and NLRAR could provide prognostic information for patients with HCC post hepatectomy. This could be valuable in guiding active individual intervention and follow-up.

\section{Ethical Approval}

This study was approved by Scientific research and New technology Institutional Review Board of Wannan Medical College Yijishan Hospital [2020-10]. Given the retrospective nature of the study without any additional interventions, the need for consent was waived by the ethics committee. All personally identifiable records were kept confidential. The guidelines outlined in the Declaration of Helsinki were followed in this study. 


\section{Funding}

This work was supported by the Wannan Medical College (WK2017F20 \& WK2017ZF09) and the Yijishan Hospital of Wannan Medical College (YR201608).

\section{Disclosure}

The authors report no conflicts of interest in this work.

\section{References}

1. Bray F, Ferlay J, Soerjomataram I, et al. Global cancer statistics 2018: GLOBOCAN estimates of incidence and mortality worldwide for 36 cancers in 185 countries. CA Cancer J Clin. 2018;68:394-424.

2. Forner A, Reig M, Bruix J. Hepatocellular carcinoma. Lancet. 2018;391:1301-1314.

3. Zhao WJ, Zhu GQ, Wu YM, et al. Comparative effectiveness of radiofrequency ablation, surgical resection and transplantation for early hepatocellular carcinoma by cancer risk groups: results of propensity score-weighted analysis. Onco Targets Ther. 2019; 12:10389-10400.

4. Nkengurutse G, Tian F, Jiang S, et al. Preoperative predictors of biochemical recurrence-free survival in high-risk prostate cancer following radical prostatectomy. Front Oncol. 2020;10:1761.

5. Guthrie GJK, Charles KA, Roxburgh CSD, et al. The systemic inflammation-based neutrophil-lymphocyte ratio: experience in patients with cancer. Crit Rev Oncol Hematol. 2013;88:218-230.

6. Yang YT, Jiang JH, Yang HJ, et al. The lymphocyte-to-monocyte ratio is a superior predictor of overall survival compared to established biomarkers in HCC patients undergoing liver resection. Sci Rep. 2018;8:2535.

7. Yamamura K, Sugimoto $H$, Kanda $M$, et al. Comparison of inflammation-based prognostic scores as predictors of tumor recurrence in patients with hepatocellular carcinoma after curative resection. J Hepatobiliary Pancreat Sci. 2014;21:682-688.

8. Zheng J, Cai J, Li H, et al. Neutrophil to lymphocyte ratio and platelet to lymphocyte ratio as prognostic predictors for Hepatocellular carcinoma patients with various treatments: a meta-analysis and systematic review. Cell Physiol Biochem. 2017;44:967-981.

9. Xia LJ, Li W, Zhai JC, et al. Significance of neutrophil-tolymphocyte ratio, platelet-to-lymphocyte ratio, lymphocyte-tomonocyte ratio and prognostic nutritional index for predicting clinical outcomes in T1-2 rectal cancer. BMC Cancer. 2020;20:208.

10. Zhou W, Kuang T, Han X, et al. Prognostic role of lymphocyte-tomonocyte ratio in pancreatic neuroendocrine neoplasms. Endocr Connect. 2020;9:289-298.

11. Sheng IY, Rayman P, Wei W, et al. Blood myeloid-derived suppressor cells correlate with neutrophil-to-lymphocyte ratio and overall survival in metastatic urothelial carcinoma. Target Oncol. 2020;15: 211-220.

12. Chen LC, Li SH, Lo CM, et al. Platelet-to-lymphocyte ratio is an independent prognosticator in patients with esophageal squamous cell carcinoma receiving esophagectomy. J Thorac Dis. 2019;11:45 $83-4590$.
13. Zhao Q, Chen S, Feng JF. A novel inflammation-based prognostic index for patients with esophageal squamous cell carcinoma: neutrophil lymphocyte ratio/albumin ratio. Oncotarget. 2017;8:1035 35-103542.

14. Zheng HL, Lu J, Xie JW, et al. Exploring the value of new preoperative inflammation prognostic score: white blood cell to hemoglobin for gastric adenocarcinoma patients. BMC Cancer. 2019; 19:1127.

15. Amin MB, Greene FL, Edge SB, et al. The Eighth Edition AJCC Cancer Staging Manual: continuing to build a bridge from a population-based to a more "personalized" approach to cancer staging. CA Cancer J Clin. 2017;67:93-99.

16. Camp RL, Dolled-Filhart M, Rimm DL. X-tile: a new bio-informatics tool for biomarker assessment and outcome-based cut-point optimization. Clin Cancer Res. 2004;10:7252-7259.

17. Ringelhan M, Pfister D, O'Connor T, et al. The immunology of hepatocellular carcinoma. Nat Immunol. 2018;19:222-232.

18. Yang HJ, Guo Z, Yang YT, et al. Blood neutrophil-lymphocyte ratio predicts survival after hepatectomy for hepatocellular carcinoma: a propensity score-based analysis. World J Gastroenterol. 2016;22: 5088-5095.

19. Mano Y, Shirabe K, Yamashita YI, et al. Preoperative neutrophil-tolymphocyte ratio is a predictor of survival after hepatectomy for hepatocellular carcinoma: a retrospective analysis. Ann Surg. 2013;258:301-305.

20. Okamura Y, Sugiura T, Ito T, et al. Neutrophil to lymphocyte ratio as an indicator of the malignant behaviour of hepatocellular carcinoma. Br J Surg. 2016;103:891-898.

21. Johnson PJ, Berhane S, Kagebayashi C, et al. Assessment of liver function in patients with hepatocellular carcinoma: a new evidence-based approach-the ALBI grade. J Clin Oncol. 2015;33: $550-558$

22. Liu PH, Hsu CY, Hsia CY, et al. Prognosis of hepatocellular carcinoma: assessment of eleven staging systems. J Hepatol. 2016; 64:601-608.

23. Shen J, Wen T, Li C, et al. The prognostic prediction role of preoperative serum albumin level in patients with intahepatic cholangiocarcinoma following hepatectomy. Dig Dis. 2018;36:306-313.

24. Zhang F, Cheng F, Cao L, et al. A retrospective study: the prevalence and prognostic value of anemia in patients undergoing radiotherapy for esophageal squamous cell carcinoma. World J Surg Oncol. 2014; $12: 244$.

25. Cordella C, Luebbers HT, et al. An evaluation of the preoperative hemoglobin level as a prognostic factor for oral squamous cell carcinoma. Head Neck Oncol. 2011;3:35.

26. Finkelmeier F, Bettinger D, Köberle V, et al. Single measurement of hemoglobin predicts outcome of HCC patients. Med Oncol. 2014;31:806

27. Liu X, Qiu H, Huang Y, et al. Impact of preoperative anemia on outcomes in patients undergoing curative resection for gastric cancer: a single-institution retrospective analysis of 2163 Chinese patients. Cancer Med. 2018;7:360-369.

28. Shankar A, Wang JJ, Rochtchina E, et al. Association between circulating white blood cell count and cancer mortality: a population-based cohort study. Arch Intern Med. 2006;16 6:188-194. 


\section{Publish your work in this journal}

The International Journal of General Medicine is an international, peer-reviewed open-access journal that focuses on general and internal medicine, pathogenesis, epidemiology, diagnosis, monitoring and treatment protocols. The journal is characterized by the rapid reporting of reviews, original research and clinical studies across all disease areas. The manuscript management system is completely online and includes a very quick and fair peer-review system, which is all easy to use. Visit http://www.dovepress.com/ testimonials.php to read real quotes from published authors. 